This item was submitted to Loughborough's Research Repository by the author.

Items in Figshare are protected by copyright, with all rights reserved, unless otherwise indicated.

\title{
Non-uniformity of deformation in low-density thermally point bonded non- woven material: effect of microstructure
}

PLEASE CITE THE PUBLISHED VERSION

http://dx.doi.org/10.1007/s10853-010-4800-1

\section{PUBLISHER}

(C) Springer

VERSION

AM (Accepted Manuscript)

\section{PUBLISHER STATEMENT}

This work is made available according to the conditions of the Creative Commons Attribution-NonCommercialNoDerivatives 4.0 International (CC BY-NC-ND 4.0) licence. Full details of this licence are available at: https://creativecommons.org/licenses/by-nc-nd/4.0/

\section{LICENCE}

CC BY-NC-ND 4.0

\section{REPOSITORY RECORD}

Hou, Xiaonan, Memis Acar, and Vadim V. Silberschmidt. 2019. "Non-uniformity of Deformation in Low-density Thermally Point Bonded Non-woven Material: Effect of Microstructure". figshare. https://hdl.handle.net/2134/19365. 


\title{
Non-uniformity of deformation in low-density thermally point bonded non- woven material: effect of microstructure
}

\section{Xiaonan Hou • Memis, Acar • Vadim V. Silberschmidt}

\begin{abstract}
One of the most important characteristic features of a low-density thermally bonded nonwoven material is its discontinuous and non-uniform microstructure, resulting in a complicated and unstable deformation mechanism of the material. In order to estimate the effects of such microstructure on the overall mechanical properties of the non-woven material, tensile tests are carried out for specimens with different systems of marks for both two principle directions - machine direction and cross direction-with images being captured with high-speed camera. The non-uniform strain fields are analysed based on the obtained images. Discontinuous finite-element models are developed to study the deformation mechanism of nonwoven specimens in both principle directions, and the effects of the discontinuous and non-uniform fibrous network and different arrangements of bond points are analysed numerically.
\end{abstract}

\section{Introduction}

Thermally point bonded non-woven materials are manufactured by interlocking polymeric fibres using heat energy that partially melt fibres in contact points during calendaring [1]. The calendaring process use heat and high pressure applied through rollers to weld the fibre webs together [2]. One of these rollers has an engraved pattern on its surface that leads to fibre-tofibre bonding locally at the points of the intersection of the engraved pattern with the smooth roller. Both rollers are heated internally, and the induced temperatures determine the level of bonding besides the other factors such as nip pressure, throughput speed and web parameters [3]. 
Due to specific features of the manufacturing process, the material is composed of three components: random fibrous network, bond points and void areas. Hence, in the literatures, the non-woven fabric is described as an anisotropic, nonhomogeneous, discontinuous and highly nonlinear material [4-14]. Although many efforts have been made to study the material's mechanical properties, there are only few works, focusing on the effects of material's non-uniform and discontinuous microstructure on them. Kim and Pourdeyhimi [15] successfully captured images using their tensile testing/image acquisition device, which showed clearly the discontinuous and non-uniform microstructure of both undeformed and deformed non-woven specimens. Gautier et al. [16] studied the strain field in the middle of non-woven specimens based on the results of optical measurements. Although their results revealed the unique deformation mechanism of non-woven materials with discontinuous and non-uniform microstructure, there is no detailed analysis of the effects of features of the material's microstructure such as fibres' orientation distribution and arrangement of bond points on the overall mechanical properties.

Mueller and Limen $[17,18]$ developed numerical models, in order to describe non-woven materials by introducing the discontinuous fibrous network and bond points. Those models were based on the use of unit cells presupposing the model's periodic structure. Hence, they cannot properly represent fibres' areal orientation distribution and various lengths of fibres. Especially, their models cannot represent non-uniform density of the fabrics. Therefore, a series of tensile tests was carried out, coupled with image analysis using specimens with different systems of marks. The obtained experimental results are used to elucidate the effects of the material's discontinuous and non-uniform microstructure on strain distribution of the material are analysed. The discontinuous finite-element (FE) models, developed in our earlier papers [19], [20], are used to describe the deformation mechanism of the low-density thermally bonded non-woven material with account for the main features of material's microstructure-a random fibrous network and a pattern of bond points. 


\section{Experimental analysis of non-uniform strain distribution}

Based on the images obtained using optical microscopy with background light (Fig. 1), the microstructure of a low-density thermally point boned non-woven material (fibre: polypropylene; diameter of fibres: $0.02 \mathrm{~mm}$; length of fibres: $30-40 \mathrm{~mm}$; density of web: 20 gsm) can be defined as highly discontinuous and non-uniform. It is easy to conclude that such a microstructure could cause a non-uniform strain distribution in the material, even for uniform external loading conditions.

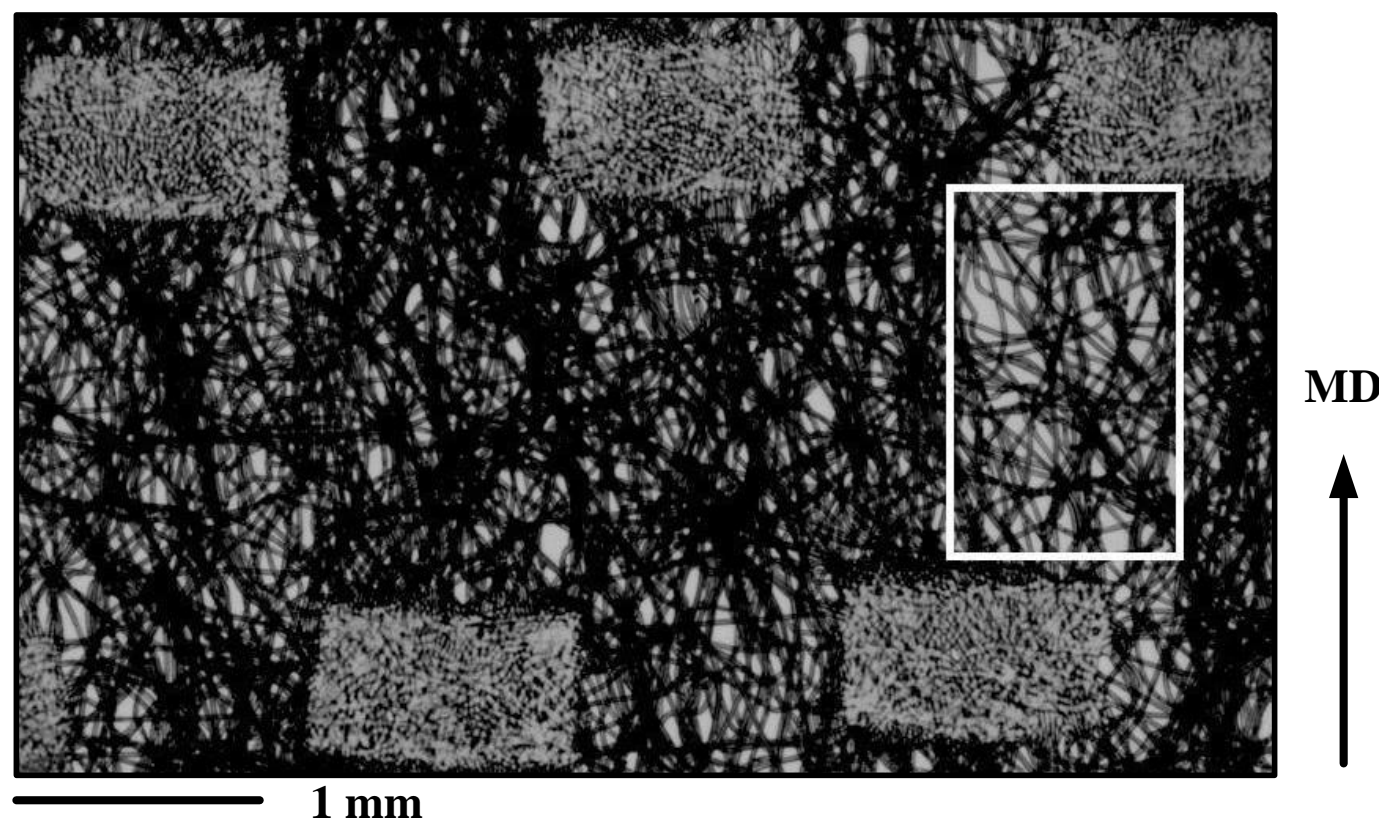

Figure 1: Non-uniform and discontinuous microstructure of nonwoven material; area with lower density is marked with rectangle

In order to analyse deformation mechanisms of the material, tensile tests were carried out coupled with image analysis. The testing area of the specimens was $25 \mathrm{~mm} 920 \mathrm{~mm}$ and the testing speed was $25 \mathrm{~mm} / \mathrm{min}$. Three systems of marks were employed for those specimens: specimen without marks, highlighted bond points and randomly highlighted points (Fig. 2). For the specimens with highlighted bond points, the marks were applied manually using a pen with black ink. For the specimens with randomly highlighted points, a random pattern of marks was applied by spraying black ink on the specimens, a method traditionally used with this technique. 


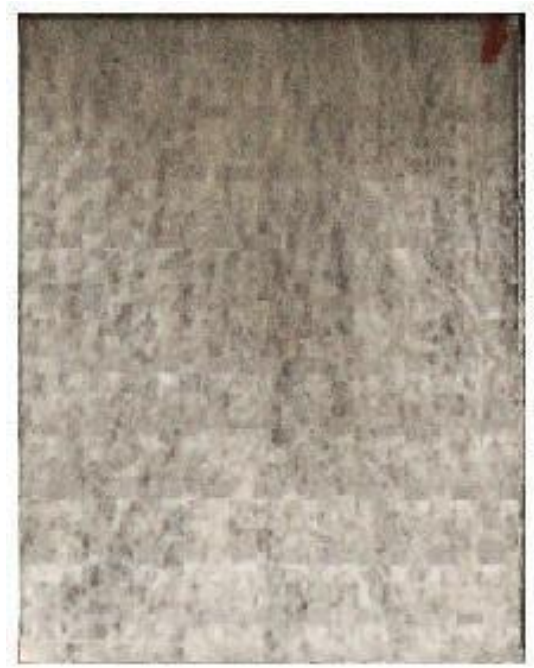

(a)

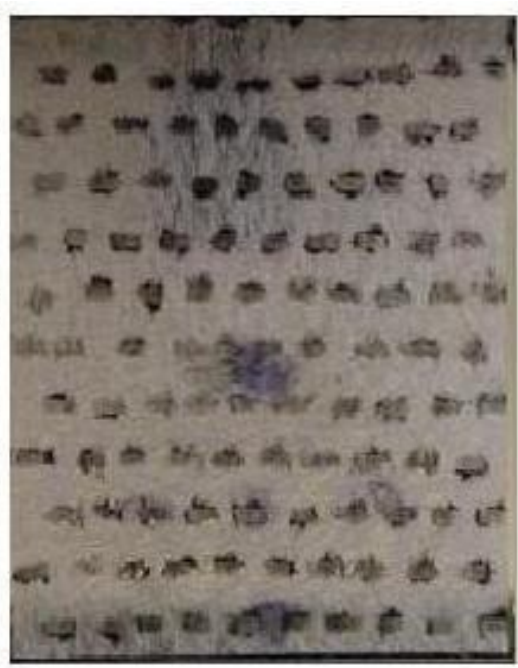

(b)

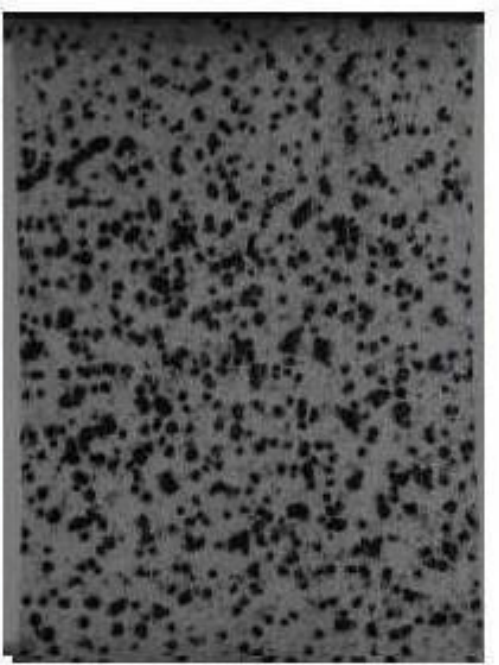

(c)

Figure 2: Specimens of nonwoven material used for image analysis: (a) specimen without marks; (b) specimen with highlighted bond points; c) specimen with random points

The specimen without any marks were tested in both machine direction (MD) and cross direction (CD) until rupture and the respective stress-strain relationship were obtained. INSTRON Micro-Tester 5848 was used for the tensile tests; the specimens were extended with one end fixed and another end moving. The specimens with highlighted bond points were tested in both MD and CD under the same conditions as specimens without marks. Images were captured with the digital camera when the strain of the specimen reaches 0,20 , 40,60 and $80 \%$. During the experiment, the height of the camera was controlled to keep the focus point targeting at the central point of the specimen to perform measurements. The specimens with randomly highlighted points were analysed with the ARAMIS system (Fig. 3), which is a 3D optical deformation analysis system for measuring deformation and strain during loading of complex materials and geometries. During the tensile test, two series of images were captured by two high-speed digital cameras and were analysed with an image analysis program provided with the system. Then strain distributions in the tested specimen were calculated for increasing strain levels. 


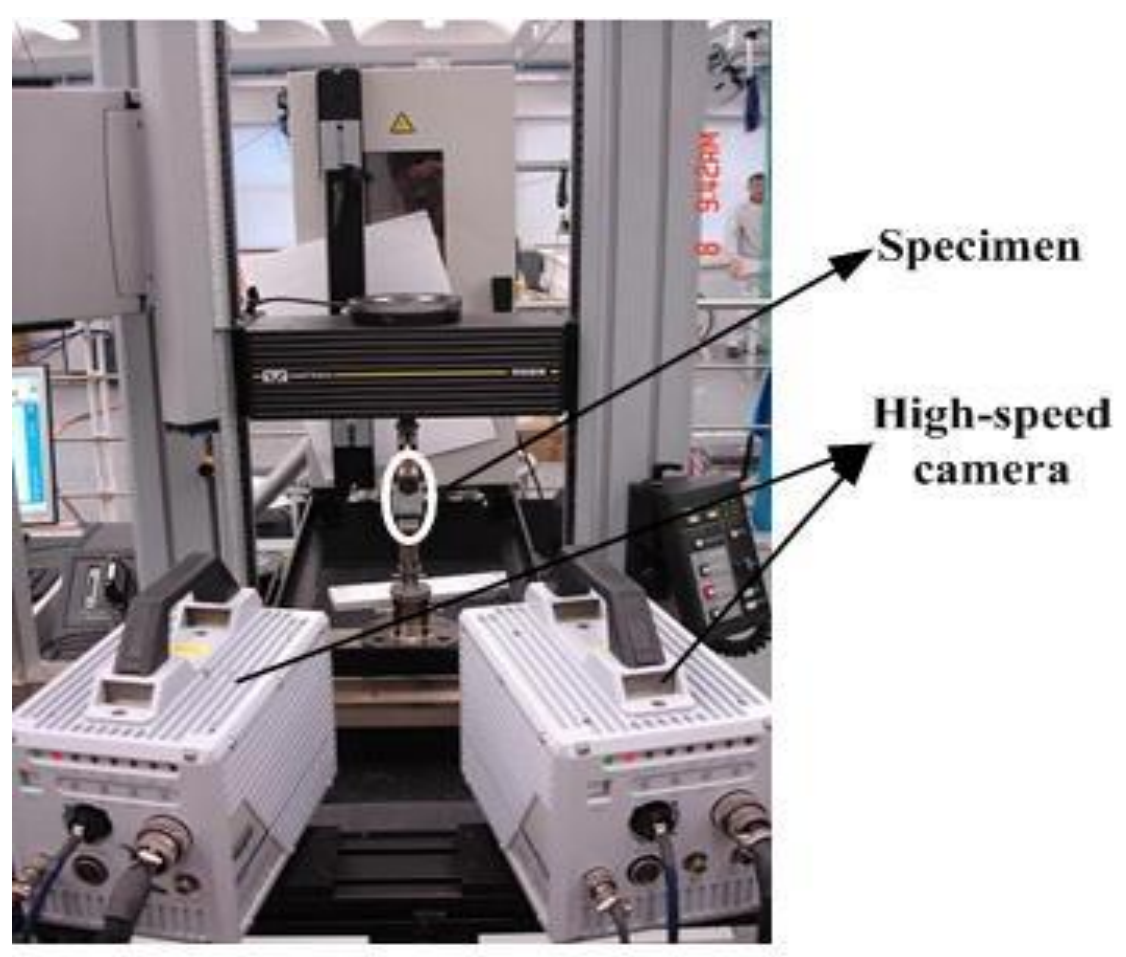

Figure 3: ARAMIS system and INSTRON testing machine

The typical stress-strain relationship of the non-woven material is shown in Fig. 4 for both $M D$ and $C D$. The MD specimen behaves almost bi-linearly; two trend lines, corresponding to various deformation stages, are drawn to fit the stress-strain curve at its elastic and plastic stages, which describes the deformational behaviour of the nonwoven material. At the breaking stage, the stress drops in two stages, which present the failure mechanism of the material. 


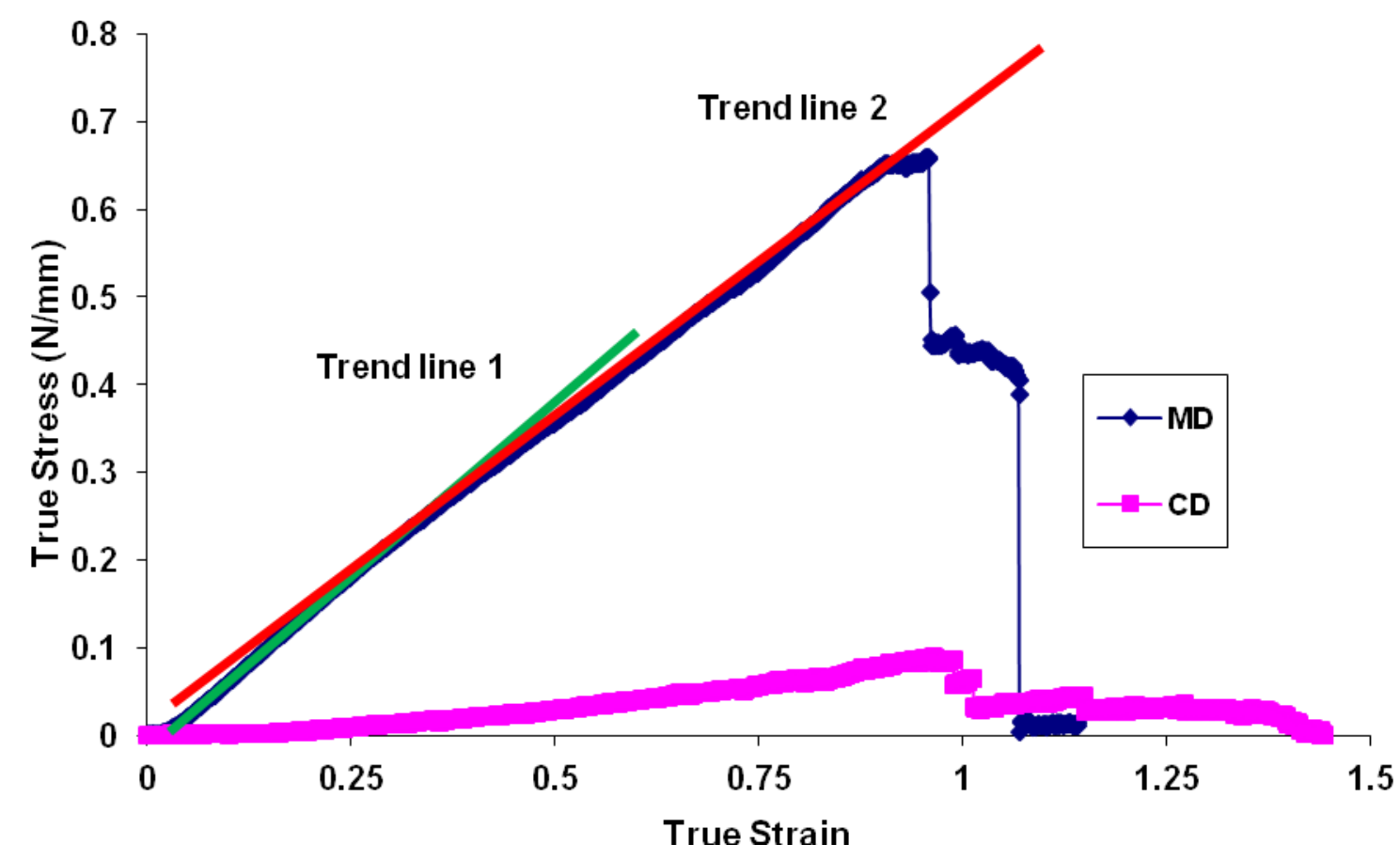

Figure 4: Comparison of typical stress-strain relationships of thermally bonded nonwoven material in machine direction and cross direction

In the MD, according to the results obtained for the specimens with highlighted bond points, at the initial stage of the tensile deformation (shown in Fig. $5 \mathrm{a}, \mathrm{b}$ ), the marks present a clear striped system, which is formed by strips of bond points with intermediate strips of the fibre network. These strips are parallel to each other. As shown in Fig. 6, the width of bond points is larger than its height, and spaces between bond points are relatively narrow (about $1 \mathrm{~mm}$ ) so when bond points form a straight line, the local material properties vary significantly between the specimen's bonding lines and the surrounding network. At the initial stage of the deformation, the "striped system" performs stably and linearly, which is represented by trend line 1 in Fig. 4 . 


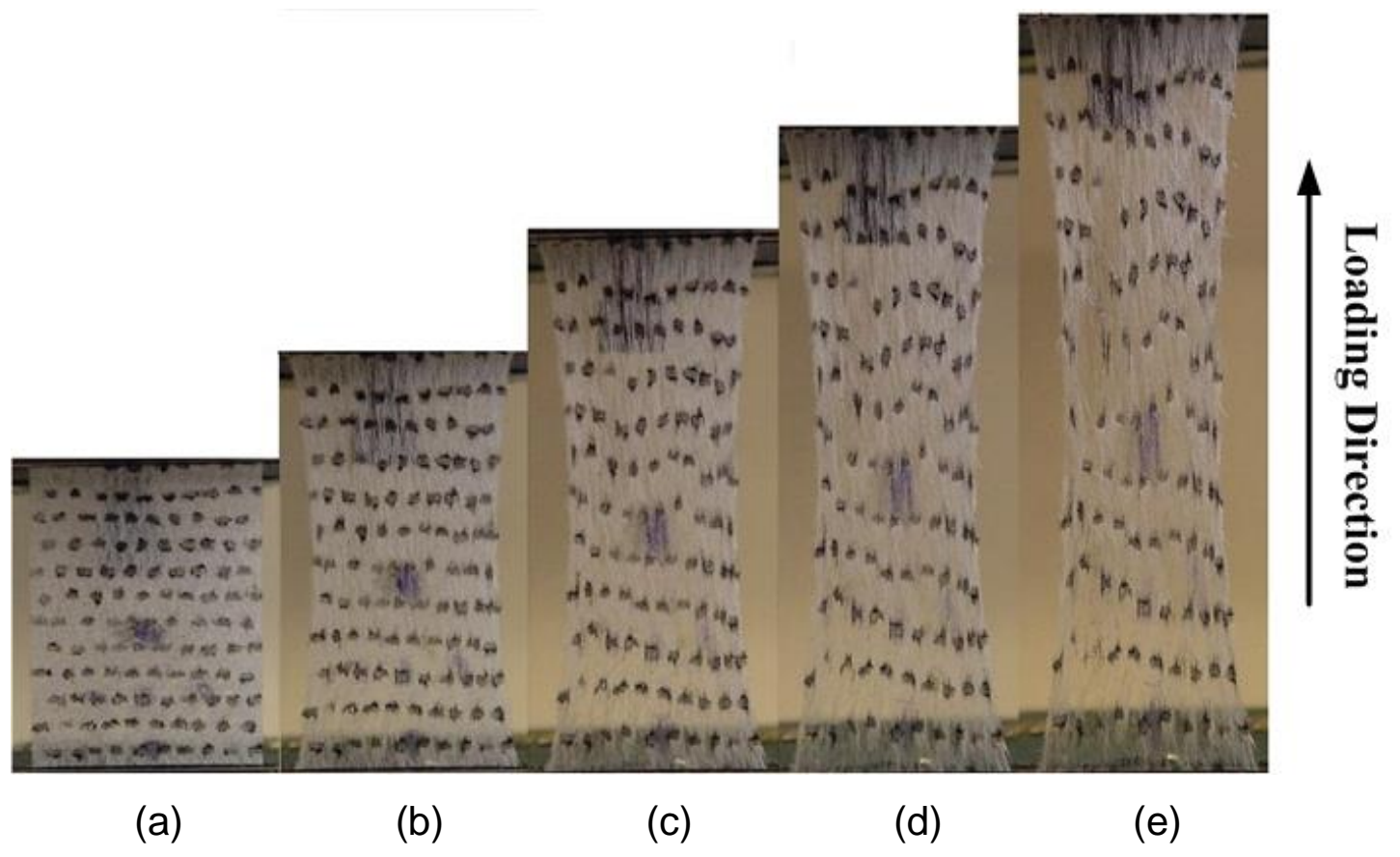

Figure 5: MD specimens with highlighted bond points: (a): non-deformed specimen; (b) specimen under $20 \%$ strain; (c) specimen under $40 \%$ strain; (d) specimen under $60 \%$ strain; (e) specimen under $80 \%$ strain

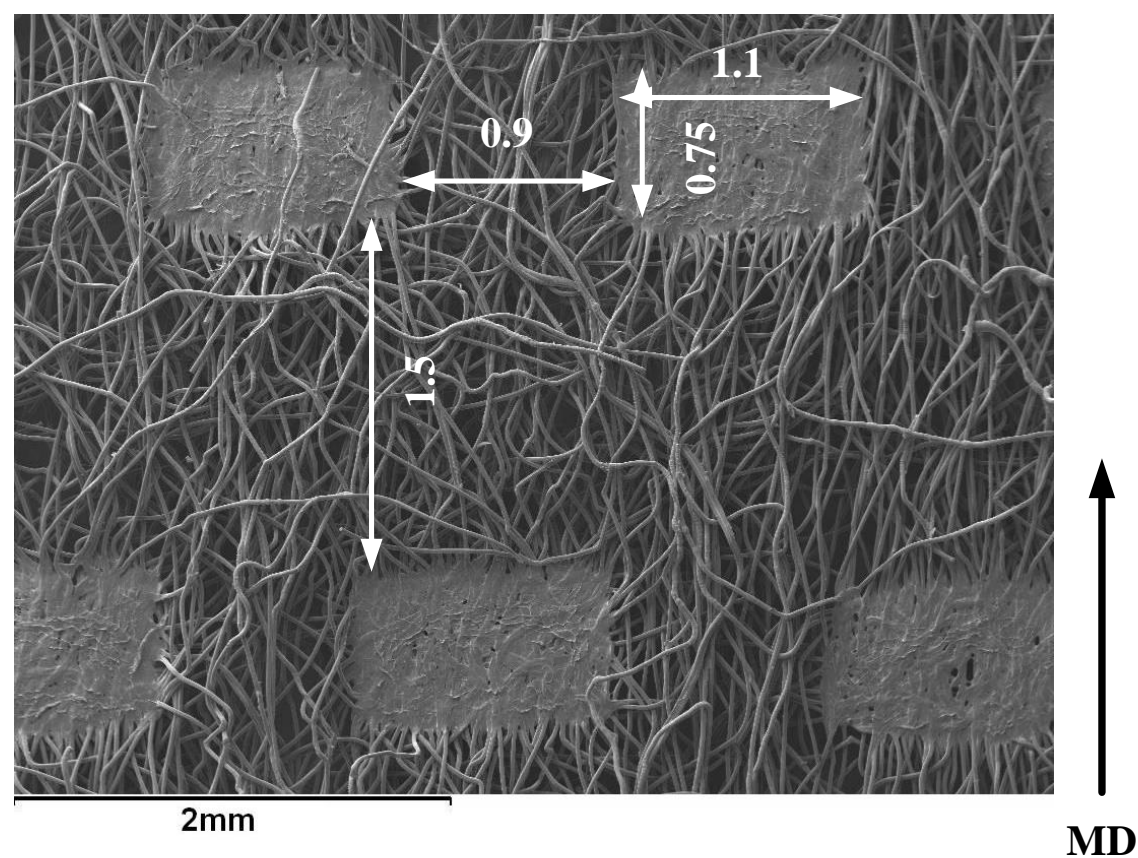

Figure 6: Dimensions of bond points and spaces between them (SEM image)

However, in the non-woven sheet, there are always local areas with a lower density as compared to other areas, due to smaller numbers of fibres being assembled in such areas 
during the manufacturing process (Fig. 1). The weaker areas are always the reason for the stress concentrations in tension and cause breaking.

Figure $5 c$ and $d$ shows deformation of the strip system at an advanced stage of the process of tension. The strips of bond points are distorted due to different local levels of stress transferred from neighbouring areas. Although, within a single strip of bond points, the spaces between bond points are narrow, the fibrous areas within the spaces still have significantly lower stiffness compared with that of the bond points. When deformation of the whole specimen achieves the advanced stage, stresses can concentrate at the areas with low density, with their stiffness even lower than that of other fibrous areas. The concentrated stress transfers from one low-density area to another, generating shear stress in the specimen and causing distortion of the strips of bond points. Moreover, the resulting distortion is not symmetric with regard to the loading direction and differs from line to line. Five specimens were tested under same conditions, and the obtained character of distortion varies from one specimen to another. The observed process vividly displays that the material deforms non-uniformly due to its discontinuous structure. The change of the deformation mechanism of the nonwoven material is responsible for the bi-linear character of the stressstrain relationship as shown in Fig. 4. When the shear stress is generated in the specimen, the slope of the stress-strain curve diminishes, resulting in the second portion of the linear behaviour, with the trend line 2 .

When the specimen is approaching its maximum strength, the material starts to break (shown in Fig. 5e), beginning from the areas with lower density. With further tensile stretching, the cracks are driven by the shear stress and transfer through the specimen linking other weak areas into a macroscopic defect.

The failure mechanism of the non-woven material is governed by both internal shear stress and external tensile stress. The rupture could start at different locations, such as the lateral boundaries of the specimen or its central area (Fig. 7). Besides a non-uniformed strain distribution caused by the boundary conditions of the experiment, the location of the rupture is determined by the location of low-density areas and the character of shear stress transfer. Moreover, the macroscopic defects develop from one area and spread until final rupture. 


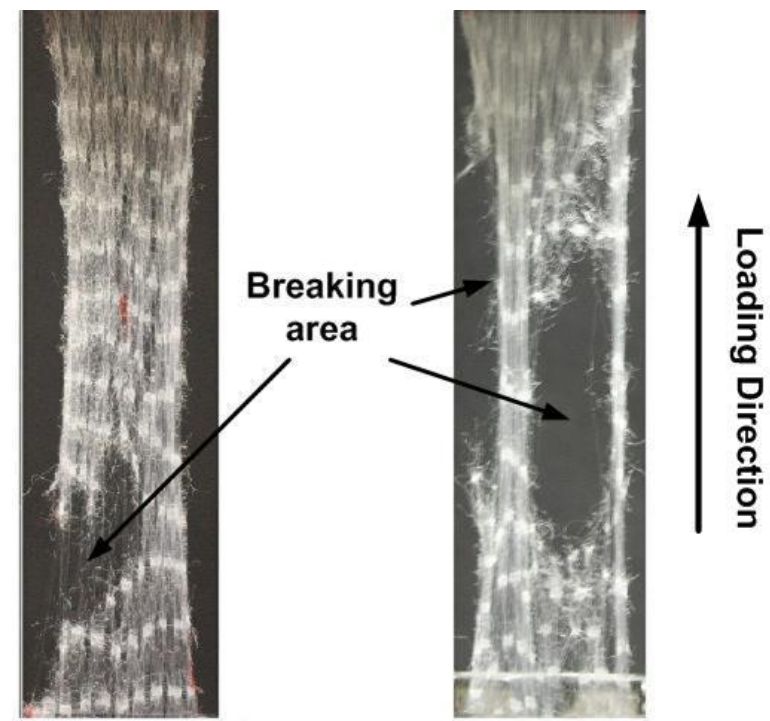

Figure 7: Different breaking areas in non-woven specimens (MD)

For the specimen with randomly highlighted points, the results obtained using the ARAMIS system for an MD specimen are shown in (Fig. 8), which gives equivalent strain distribution mapping for the deformed material. The images for strain levels of $0,20,40$ and $60 \%$ were collected to compare with each other to demonstrate the change of the strain distribution; different colours correspond to different strain levels. Figure $8 \mathrm{~b}$ displays the masked specimen with $0 \%$ strain, with a uniformly distributed dark (blue) area demonstrating the uniform state. In the specimen exposed to overall strain of $20 \%$, the material in the boundary area near grips has larger local strains-about $60 \%$, having a different shade of grey (red) in Fig. $8 \mathrm{c}$. It is due to the constrained boundary condition applied by the grips. Other areas of the specimen, away from the grips, are still practically under uniform strain. When the specimen achieves overall strain of $40 \%$ (Fig. 8d), the strain in the central parts of the specimen is distributed nonuniformly and apparently is not affected by bond points, which are located in the material regularly. In the specimen at $60 \%$ strain, the strain non-uniformity achieves a higher level, with the areas with high levels of strain located in the material randomly. The observed character of strain distribution means that the non-uniform deformation mechanism of the material is not only caused by the boundary conditions of the specimen and the bond points, which are formed by a stiffer material, causing stress concentration, but also resulted from the random discontinuous fibrous network and its random density distribution. 


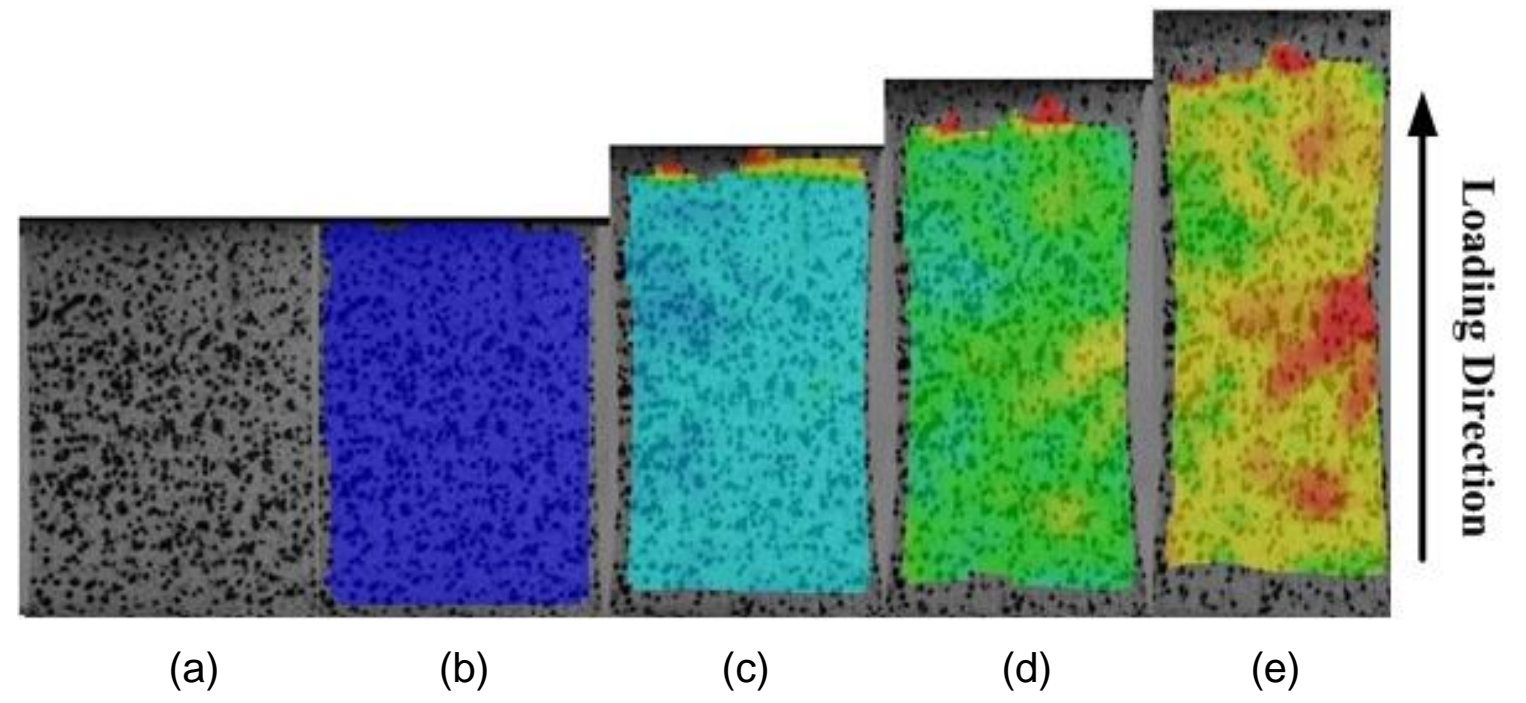

Figure 8: Results obtained with ARAMIS system for MD: a nondeformed specimen; $b$ masked specimen under $0 \%$ strain; c specimen under $20 \%$ strain; d specimen under $40 \%$ strain; e specimen under $60 \%$ strain (Color figure online)

In CD, the typical stress-strain curve of the specimens, which is obtained from the tensile tests of specimens without marks, shows a highly nonlinear behaviour before its rupture. However, it is still possible to simplify the nonlinear behaviour of the specimen to a tri-linear behaviour as shown in Fig. 9. These three trend lines are used to present different stages of the deformation.

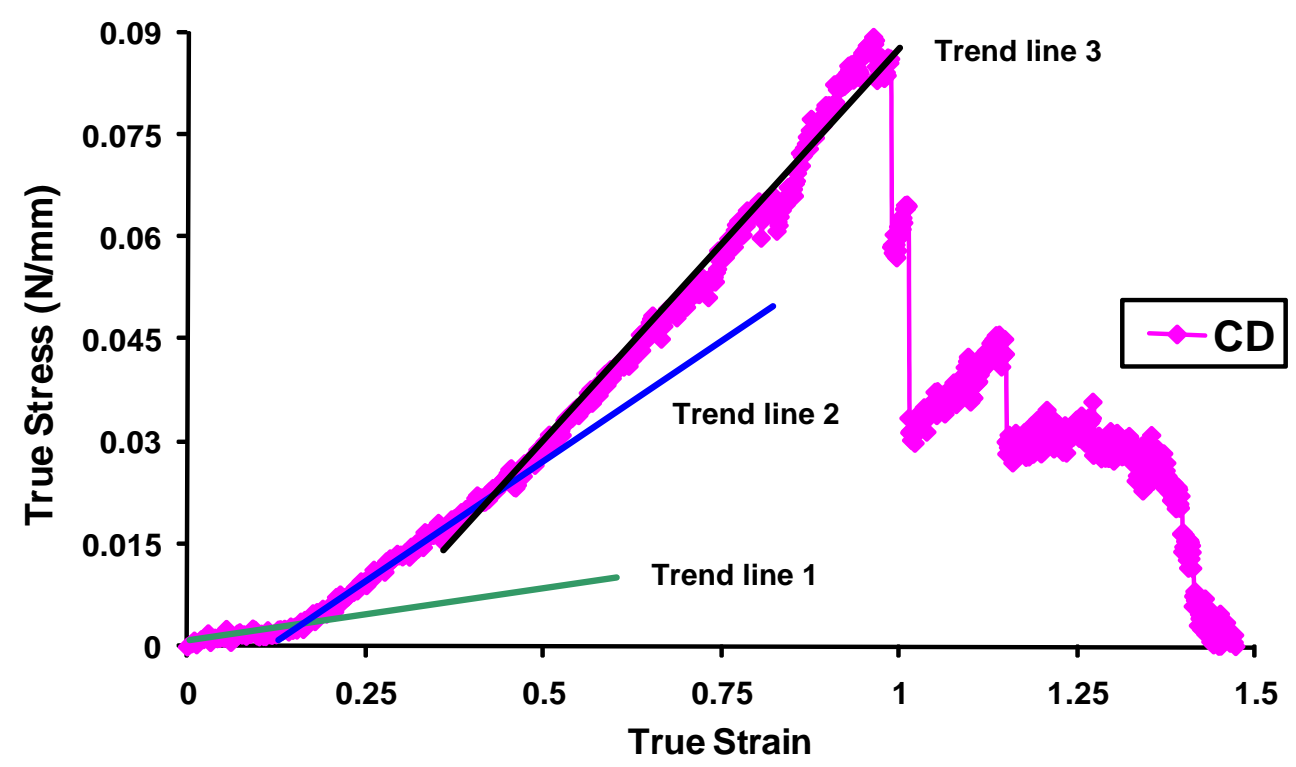

Figure 9: Typical stress-strain relationship of thermally bonded nonwoven material in cross 
direction

According to the tensile tests and the image analysis of specimens with highlighted bond points (Fig. 10), the deformation mechanism of elastic extension in the CD is more complicated, and it can be divided into three main stages.

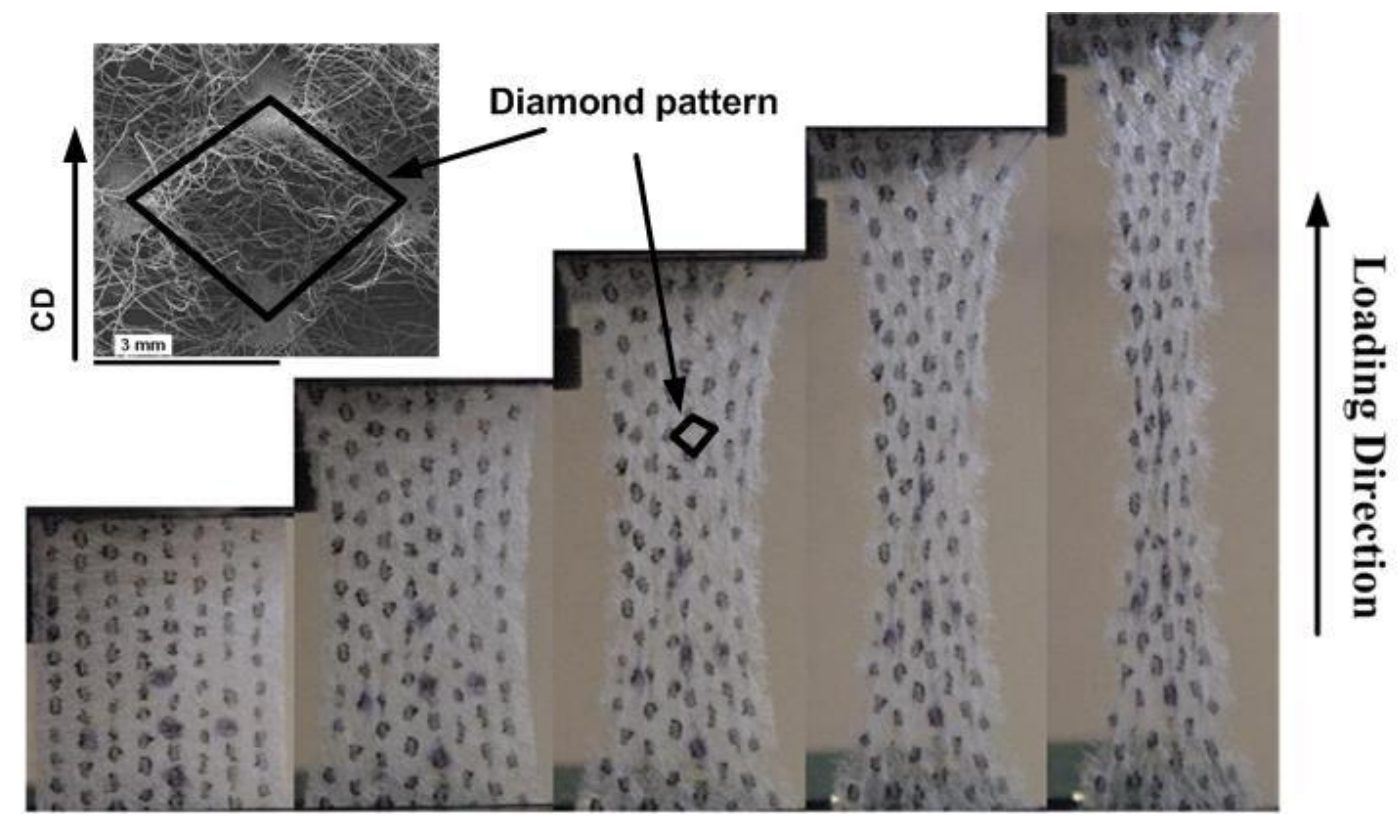

(a)

(b)

(c)

d)

(e)

Figure 10: $C D$ specimens with highlighted bond points: (a) non-deformed specimen; (b) specimen under $20 \%$ strain; (c) specimen under $40 \%$ strain; (d) specimen under $60 \%$ strain; (e): specimen under $80 \%$ strain

At the initial stage of tension (Fig. 10b), the slope of the stress-strain curve is low, which is due to the presence of fibre-net strips along the extension direction as shown in Fig. 11. The fibre-net strip consists of curly fibres with friction between fibres. Therefore, the fibre-net strips have really low stiffness. Moreover, due to the arrangement of the bond points, the space between two neighbouring points along the CD is the narrowest one. Therefore, it is obvious that the fibres connecting two neighbouring bond points along CD are easier to stretch so they start carrying the load earlier than other fibres. However, the number of fibres connecting two neighbouring bond points along the CD represents only a small part of their total number. It causes the initial stiffness (presented by trend line 1) of the specimen at this stage to be lower than the stiffness at more advanced stages of the material's deformation (trend lines 2 and 3 in Fig. 10). Therefore, at the initial stage, the deformation mechanism of 
CD specimens is mainly governed by the straightening of curly fibres, release of entangled fibres and the initial deformation of fibres between two neighbouring bond points along the CD.

With further extension, four neighbouring bond points form a diamond pattern (shown in Figs. 10c, 11). The fibres, which are connecting two neighbouring bond points along the $C D$ start to carry more load due to the larger external extension. Moreover, the diamond pattern becomes a basis for load bearing, and the stress also transfers along the boundaries of the diamonds. At the end of this stage, the fibres between two neighbouring bond points along the CD start to break. The diamond pattern begins to contract rapidly (Fig. 10d, e), and holes appear in the areas with low density of fibres inside diamond-shaped cells. This deformation stage is presented by trend line 2 in Fig. 9.

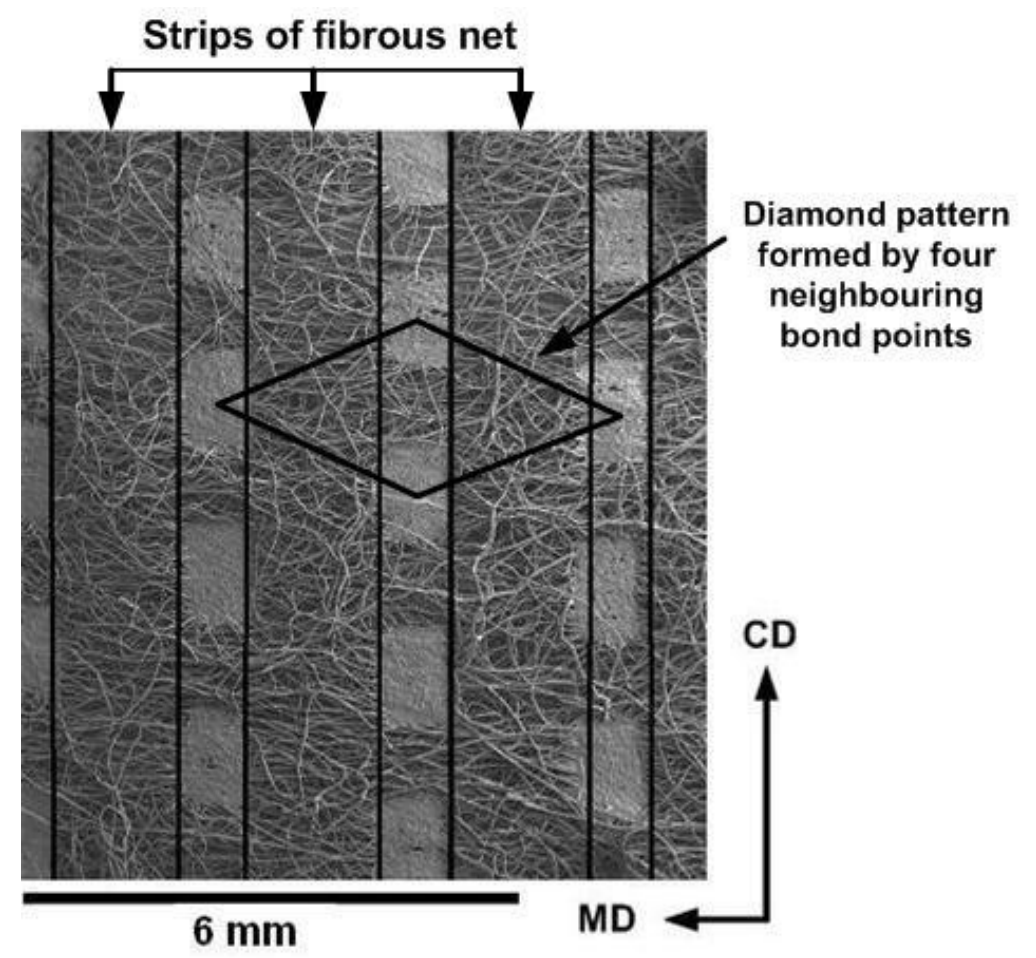

Fig. 11 Fibrous net strips along cross direction

At the following stage of the deformation process, the boundaries of diamond shapes are rearranged by longitudinal tensile stretching and lateral contraction. They are reoriented towards the loading direction (CD) and carry the load. This is shown schematically in Fig. 12. At this stage, more fibres are arranged along the loading direction due to the reorientation caused by external extension. Therefore, the material becomes stiffer, which is presented by 
trend line 3 (Fig. 9).

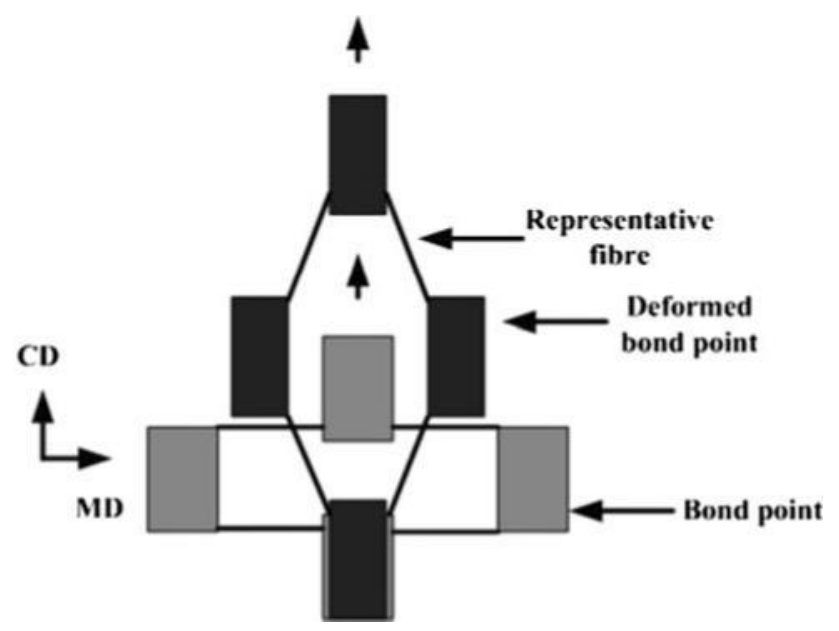

Fig. 12 Transformation of diamond pattern at advanced stage of tensile test

When the material starts to break, the initial breaking points are often found on a boundary of bonding points, which is the stress-concentration area, at the third stage of the tensile behaviour of $C D$ specimens. As shown in Fig. 13, the rupture of the material usually develops in stages due to the material's partial rupture, which is the reason for the drops in the stress on the stress-strain curve. It is similar to the phenomena found in MD specimens. At the breaking stage, the deformation mechanism of the material is dominated by the tension of bundles of fibres along the loading direction and shear stress transferring from one damage area to another one.

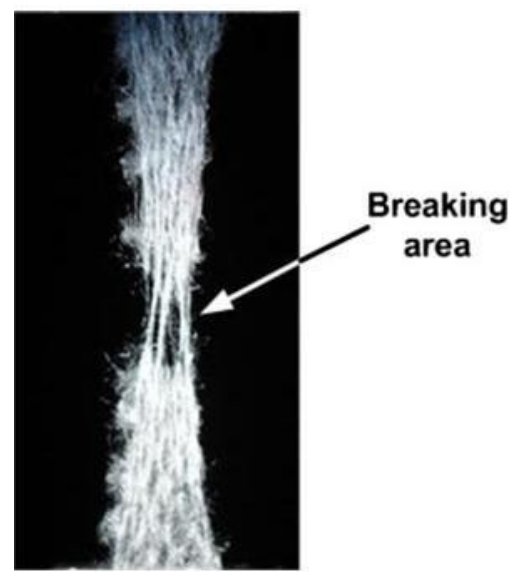

Fig. 13 Finial (breaking) tensile stage of specimen in cross direction

The analysis with the ARAMIS system for CD specimens demonstrates a highly non-uniform 
strain distribution (Fig. 14). High level of strain non-uniformity was found even for the specimen under $20 \%$ strain. It means that in the specimen stretched in the CD, strains are distributed more non-uniformly than in MD specimen (see Fig. 8), and large local deformations happen in some areas even at the very early stages of extension. When the specimen is under the overall strain of $40 \%$, some areas of the material start to achieve $60 \%$ strain [dark spots (red) in the centre of the specimen, Fig. 14d]. And for the overall specimen's deformation of $60 \%$, some areas of the material still have low-strain levels [lighter (green and yellow) areas in Fig. 14c]. These phenomena demonstrate that the material has a random, non-uniform deformation mechanism, with the level of its non-uniformity in CD higher than in MD.

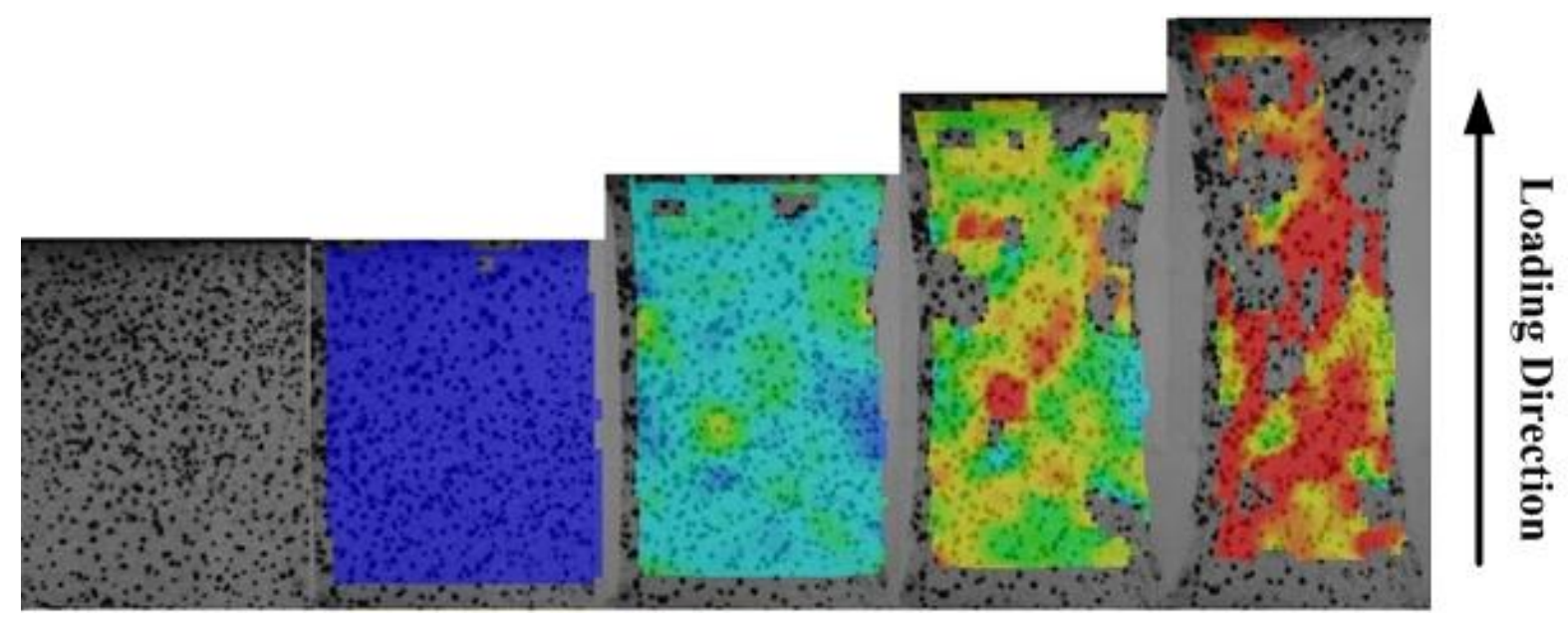

(a)

(b)

(c)

(d)

(e)

Fig. 14 Results obtained with ARAMIS system for CD: a non-deformed specimen; $b$ masked specimen under $0 \%$ strain; c specimen under $20 \%$ strain; d specimen under $40 \%$ strain; e specimen under $60 \%$ strain (Color figure online) 


\section{Numerical analysis}

In order to analyse the non-uniform deformation mechanism of the non-woven material quantitatively, FE models are developed, and the results of numerical simulations are compared with the experimental ones. The main approach to develop a discontinuous FE model is discussed in previous papers [19, 20]. The geometry part of the models consists of areas meshed with solid elements, presenting bond points, and trusses presenting the fibrous network. It was generated by assuming the fibres to be straight and arranged according to their orientation distribution function. Due to a large number of fibres, forming the real non-woven material, it is not practical and efficient to introduce their exact number into the discontinuous model. The trusses within the geometry part of the FE model are representative fibres, which represent a certain number of fibres having close directions; the effective modulus for truss elements is calculated according to the modulus of real fibres.

In order to describe the tensile behaviours of the nonwoven material for its two principle directions - MD and CD—and analyse the effects of its non-homogenous and discontinuous microstructure, two models with different arrangements of bond points and fibre's orientation distributions were developed. Two different fibrous webs were generated according to measured orientation distribution functions to represent two principle directions of the material and 245 fibres were used to model each fibrous web [20].

For OD1 distribution, the highest frequency of fibres occurs for $90^{\circ}$, the ratio of anisotropy is 1.16; a distribution with the largest number of fibres assembled along $0^{\circ}$ (loading direction), is denoted OD2, which is an inverted OD1 and the ratio of anisotropy is 0.86 [20].

Two different arrangements of bond points-staggered MD and staggered CD-were suggested for modelling [20]. The dimensions of bond points for both arrangements correspond to those of the real non-woven material. For the MD arrangement, the strips of bond points, which are formed by their lines, are perpendicular to the loading direction; it was used to simulate the real non-woven. The CD arrangement simulated the arrangement of bond points for $\mathrm{CD}$ specimens of the real material.

Two discontinuous models were developed based on the two types of fibres orientation distributions and two arrangements of bond points: OD2/staggered MD and OD1/staggered CD. The former model was developed to simulate the tensile behaviour of the real nonwoven material in MD, while the latter one was used for $C D$. In order to simulate the tensile behaviours of the non-woven fabric for two principle directions, the boundary conditions of the models were applied as follows: one edge, perpendicular to the loading direction, was 
fixed as 'ENCAS-TRE'. All the degrees of freedom of the nodes located at this boundary were constrained. This boundary condition corresponds to fixture of the specimen in a grip in the real-life test. A uniform displacement in x-direction was applied to the opposite boundary of the models to simulate the static tensile deformation.

The obtained results for discontinuous FE models demonstrate non-uniform stress-strain distributions in the non-woven material, due to the random and discontinuous microstructure of the models [20]. First, the models reveal that the fibres in the deformed fabric are at different strain levels. Some of the fibres, in different locations and with different directions, are at lower strain levels than that of the overall material, while some of them are at higher strains. Since all the fibres in the non-woven fabric are assumed to have same mechanical properties, the strain level of fibres determines their stress. It is therefore the fibres within one fabric carry different levels of stress during the overall deformation the fabric.

Second, the effect of arrangement of bond points on the deformation mechanism of the material is also described by the model. The models with the staggered MD arrangement of bond points deform as a striped system formed by lines of bond points with intermediate layers of the fibrous web. However, due to the non-uniformed areal density, the striped system starts to distort at a relatively higher strain level. This finding agrees with the results of our experimental analysis presented in the previous section. For the models with the staggered CD arrangements of bond points, the initial deformation behaviour is based on a generation of the diamond pattern, formed by four neighbouring bond points. Fibres connecting two neighbouring bond points along the loading direction carry higher stresses even at relatively low overall strain levels, due to their orientation and smaller initial length. It is therefore can be anticipated that these fibres tend to rupture at early stages of the deformation process in the real test. This will make the diamond patterns the basic load carrier, with the load transferred by the boundaries of diamond-shaped cells. These phenomena also agree to the experimental observations. The finding is essential to understand the non-uniform mechanical performance of the material and provides important information for the analysis of its failure mechanism. Figure 15 demonstrates quantitatively the strain distribution in fibres in the deformed FE models, when the overall models are under different strain levels. And Table 1 reveals the magnitudes of average strains of the fibres for the analysed FE models. 


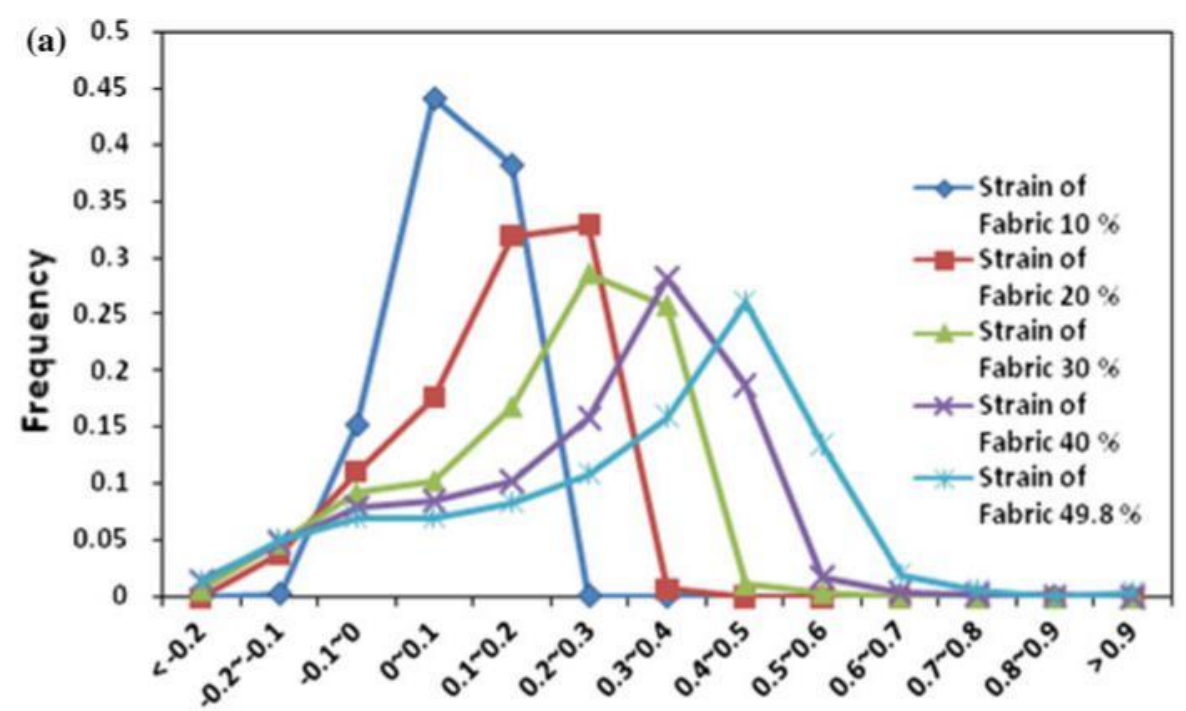

Strain of Fibres

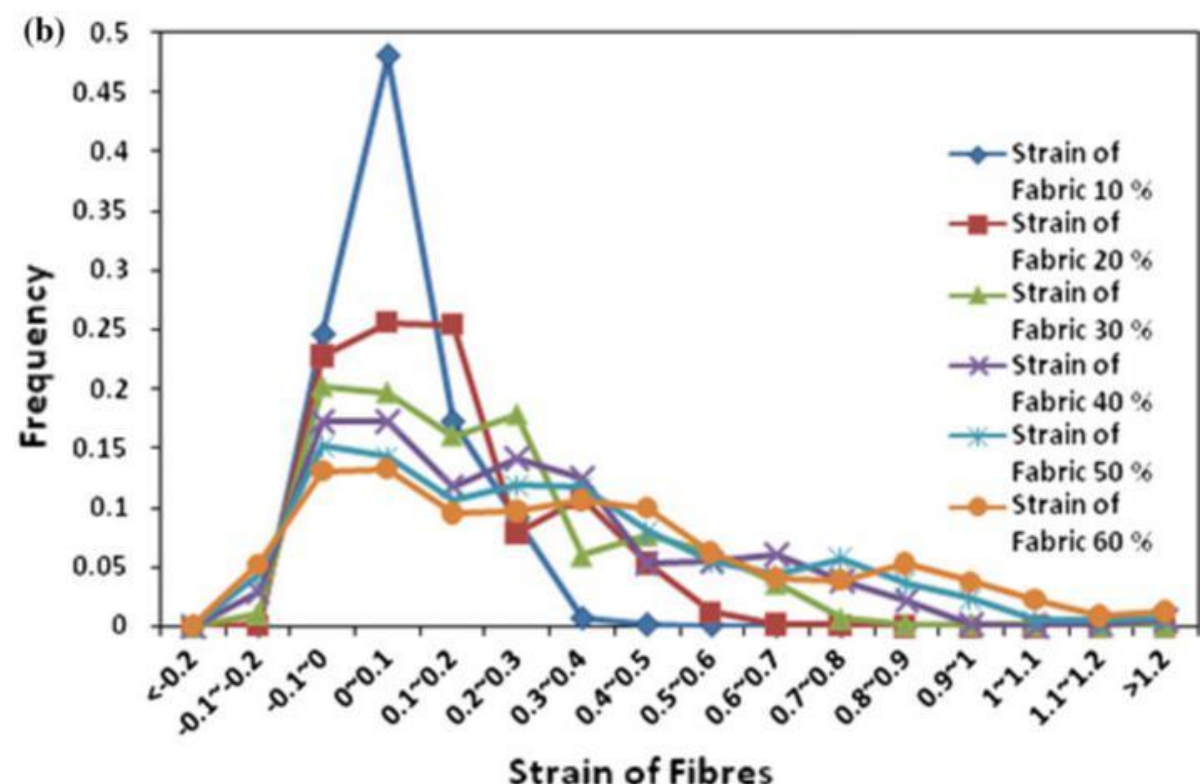

Fig. 15 Distribution of strain of fibres MD model (a) and CD model (b)

\begin{tabular}{|c|c|c|c|c|c|}
\hline \multirow{2}{*}{$\begin{array}{l}\text { Strain of } \\
\text { fabric }(\%)\end{array}$} & \multicolumn{2}{|l|}{ MD } & \multirow{2}{*}{$\begin{array}{l}\text { Strain of } \\
\text { fabric }(\%)\end{array}$} & \multicolumn{2}{|l|}{$\mathrm{CD}$} \\
\hline & $\begin{array}{l}\text { Average strain } \\
\text { of fibres }(\%)\end{array}$ & $\begin{array}{l}\text { Standard } \\
\text { deviation }(\%)\end{array}$ & & $\begin{array}{l}\text { Average strain } \\
\text { of fibres }(\%)\end{array}$ & $\begin{array}{l}\text { Standard } \\
\text { deviation }(\%)\end{array}$ \\
\hline 10 & 6.6 & 5.8 & 10 & 7.0 & 8.5 \\
\hline 20 & 13.0 & 10.8 & 20 & 13.4 & 15.7 \\
\hline 30 & 19.0 & 15.2 & 30 & 19.4 & 21.8 \\
\hline 40 & 24.8 & 19.0 & 40 & 24.9 & 28.0 \\
\hline 49.8 & 30.3 & 22.3 & 50 & 29.9 & 33.2 \\
\hline - & - & - & 60 & 34.5 & 37.7 \\
\hline
\end{tabular}

Table 1 Effect of overall strain on average strain in fibres and its standard deviation 
The strain distribution in fibres for the MD model is given in Fig. 15a, clearly demonstrating its non-uniform character. For instant, when the overall strain of the model is $10 \%$, the maximum strain for the fibres in the model is $22.1 \%$, the minimum strain of fibres is $-12.6 \%$ (i.e. in compression). However, the average strain of fibres is $6.6 \%$, which is lower than overall strain. It is due to the fact that the fibres located at the corners of the model tend to have extreme strain levels due to the effect of lateral contraction. The fibres, aligned perpendicular to the loading direction, are normally compressed, having negative strain for an overall stretching because of this contraction. With the increase in the overall strain, the distribution changes and the axial strains of fibres increase. However, the average strains of fibres are constantly smaller than overall strain (Table 1). The reason for this is that elongation of the model is caused not only by elongation of fibres but also by changes in the microstructure of the model, resulted from the reorientation of the fibres. The extent of strain non-uniformity also increases, when the overall strain achieves a higher level, as shown, by the increasing standard deviation in Table 1. Although the proportion of compressed fibres in the MD model slightly decreases-from 15.5 to $13.5 \%$-with the increase in the overall strain-from 10 to $49.8 \%$-it basically stays at a similar level. It means that the number of compressed fibres is generally determined by the original microstructure of the material and is not affected significantly by stretching of the fabric up to rather high level. Obviously, the small decrease is due to the reorientation of fibres.

For the CD model, the fibres' strain distribution is shown in Fig. 15b. The results demonstrate that the character of this distribution is different from that for the MD model, due to the different arrangements of bond points and fibre's orientation distributions. The average strains of fibres for the CD model are similar to those for the MD model (Table 1). However, as revealed by the analysis, the standard deviations of strain of fibres for the CD model are generally higher than the one for the MD model. This means that the nonuniformity level of the CD model is much higher. Hence, the fibres in the CD model have a higher risk to achieve extremely higher strain levels. Regarding the fraction of the compressed fibres in the CD model, it steadily decreased from $24.6 \%$ at the overall strain of $10 \%$ to $18.6 \%$ at $60 \%$, in a similar way to the in MD model. 


\section{Conclusions}

In order to investigate experimentally the effects of a nonuniform microstructure on the deformation mechanism of the non-woven material, tensile tests and image analysis were used. From the images of non-woven material's microstructure, it is obvious that it should be treated as a three-component material with a highly non-uniform structure. The latter is the main factor that determines a non-uniform deformation character of the material.

According to the obtained experimental results, the deformation mechanism of the material is affected by the arrangement of bond points and its non-uniform microstructure. For the MD, the specimen presents clearly a 'striped system', which is formed by strips of bonding points with intermediate strips of the fibrous network. At the initial stage of deformation, the striped system behaves stably and linearly. When the specimen achieves advanced strain levels, the strips of bond start to distort; it is caused by the nonuniform structure of the material. The stress concentrates at the areas with a low local density and transfers from one low-density area to another, generating shear stress in the specimen. Finally, a micro-failure occurs starting from a low-density area, and the crack propagates though the specimen forming a macroscopic defect. For the $C D$, due to the arrangement of bond points and fibres' orientation distributions, the level of non-uniform strain is higher than for the MD, and the deformation mechanism is more complex. At the initial stage, deformation is governed by stretching of curly fibres in fibre-net strips along the loading direction and initial deformation of fibres, orientated along, or close to deformation direction, between two neighbouring bond points. With further extension, four neighbouring bond points form a cell of a diamond pattern. The fibres connecting two neighbouring bond points along the CD mainly carry the load until they start to break, forming holes inside the cells of the diamond pattern. At this stage, the pattern begins to contract rapidly in the lateral direction with increasing strain. The boundaries of diamond-shaped cells are rearranged by longitudinal tensile stretching and lateral contraction to align along the loading direction. Finally, an initial breaking point is usually found on a boundary of a bonding point, starting rupture.

The results of numerical simulations revealed that the material's non-homogenous threecomponent structure did affect its deformation mechanism and cause non-uniform distributions of stresses and strains for deformation along its both principle directions. The obtained features of deformation mechanisms agree with the experimental observation. For both MD and CD of the material, the strain level in fibres increases with the increase of overall strain. However, due to the random and discontinuous fibrous network, the average strain of fibres is always lower 
than the overall strain of fabric. The level of non-uniformity of the strain distribution also increases with the tension process, with the CD specimen generally demonstrating higher levels of non-uniformity than the MD one.

According to both the experimental and numerical results, the reasons for the non-uniform strain distribution of the non-woven material can be summarised in the following way:

The non-uniform strain distribution in the material is caused by the manufacturing induced non-homogenous and discontinuous microstructure. The local density of the fibrous network fluctuates, leading to a non-uniform strain-stress distribution during the deformation. Its areas with low density usually cause local failure and initiate the final rupture of the overall material at higher strain levels. Beside, the fibres within the fabric are assigned in different directions, when the overall fabric is under a uniaxial loading, different fibres are under different strain levels from the overall strain.

The arrangement of bond points has an additional effect. The stiffness of bond points is higher than that of the fibrous network, causing stress concentration. Therefore, the arrangement and shape of bond points affect the deformation mechanism of the material. A character of boundary conditions, reflecting the real-life high-extension experiments with the non-woven also affects the extent of non-uniformity of deformation, but this factor would be the same for a uniform material.

Acknowledgements We greatly acknowledge the support by the Nonwovens Cooperative Research Center of North Carolina State University, Raleigh, USA.

\section{References}

1. Krčma R (1962) Nonwoven textiles, 1st edn. SNTL, Manchester

2. Erel S, Warner SB (2001) Text Res J 71:22

3. Bhat GS, Malkan SR (2002) J Appl Polym Sci 83:572

4. Petterson DR, Backer S (1963) Text Res J 33:809

5. Hearle JWS, Stevenson PJ (1963) Text Res J 33:877

6. Kim HS, Pourdeyhimi B, Desai Pet al (2001) Text Res J 71:965

7. Russell SJ (ed) (2007) Handbook of nonwovens, 1st edn. Woodhead Publishing Limited 
and CRC Press LLC, Boca Raton, FL

8. Rawal A, Priyadarshi A, Lomov SV et al (2010) J Mater Sci 45:2274. doi:10.1007/s10853009-4152-x

9. Kang KY, Lee KY, Jo KJ, Kim HS (2008) J Mater Sci 43:2754. doi:10.1007/s10853-0082482-8

10. Termonia Y (1997) Chem Eng Sci 52:3003

11. Rawal A, Lomov S, Ngo T et al (2007) Text Res J 77:417

12. Rawal A, Lomov S, Verpoest I (2008) J Text Inst 99:235

13. Rawal A (2006) J Ind Text 36:133

14. Kim HS, Pourdeyhimi B (2001) J Text Appeal Technol Manag 1:1

15. Kim HS, Pourdeyhimi B, Abhiraman AS (2001) Text Res J 71:157

16. Gautier KB, Kocher ChW, Drean J (2007) Text Res J 77:20

17. Mueller DH, Kochmann M (2004) Int Nonwovens J 13:56

18. Limem S, Warner SB (2005) Text Res J 75:63

19. Hou X, Acar M, Silberschemidt VV (2009) Comput Mater Sci 46:700

20. Hou X, Acar M, Silberschemidt VV (2010) Finite element simulation of low-density thermally bonded nonwoven materials: effects of orientation distribution function and arrangement of bond points. Comput Mater Sci. doi: 10.1016/j.commatsci.2010. 03.009) 